\title{
Severe Gastrointestinal Disorder due to Capecitabine Associated with Dihydropyrimidine Dehydrogenase Deficiency: A Case Report and Literature Review
}

\author{
Yuya Hagiwara ${ }^{1}$, Yoshiyuki Yamamoto ${ }^{1}$, Yuki Inagaki ${ }^{2}$, Reina Tomisaki ${ }^{1}$, Miki Tsuji ${ }^{1}$, \\ Soma Fukuda ${ }^{1}$, Satoshi Fukuda ${ }^{1}$, Tsubasa Onoda ${ }^{1}$, Hirosumi Suzuki ${ }^{1}$, Yusuke Niisato ${ }^{1}$, \\ Yoshitaka Tange ${ }^{1}$, Naoya Ikeda ${ }^{2}$, Keiichi Yamada ${ }^{2}$, Mariko Kobayashi ${ }^{1}$, Daisuke Akutsu ${ }^{1}$, \\ Takeshi Yamada ${ }^{1}$, Toshikazu Moriwaki ${ }^{1}$, Toshiaki Narasaka ${ }^{1}$, \\ Hideo Suzuki ${ }^{1}$ and Kiichiro Tsuchiya ${ }^{1}$
}

\begin{abstract}
:
Dihydropyrimidine dehydrogenase (DPD) deficiency induces severe adverse events in patients receiving fluoropyrimidines. We encountered a 64-year-old DPD-deficient man with a severe capecitabine-related gastrointestinal disorder. He received capecitabine-containing chemotherapy after rectal cancer resection. During the first course of chemotherapy, he developed severe diarrhea, a fever, and hematochezia. Endoscopy revealed mucosal shedding with bleeding throughout the gastrointestinal tract. DPD deficiency was suspected because he developed many severe adverse events of capecitabine early and was finally confirmed based on the finding of a low DPD activity level in peripheral blood mononuclear cells. After one month of intensive care, hemostasis and mucosal healing were noted, although his gastrointestinal function did not improve, and he had persistent nutritional management issues.
\end{abstract}

Key words: dihydropyrimidine dehydrogenase (DPD) deficiency, capecitabine, adverse event, gastrointestinal bleeding, gastrointestinal disorder

(Intern Med Advance Publication)

(DOI: 10.2169/internalmedicine.8636-21)

\section{Introduction}

Fluoropyrimidine (FP) anticancer agents are commonly used in daily clinical practice as key drugs for gastrointestinal, breast, and head and neck cancers. Dihydropyrimidine dehydrogenase (DPD) is the first rate-determining enzyme in the catabolism of pyrimidine bases uracil and thymine (1) and is also known as an important enzyme that catalyzes the metabolic degradation of FPs (2). Patients with deficiency or reduced activity of DPD are prone to serious adverse events, and administration of FPs to such patients may even lead to death.

We herein report a case of DPD deficiency in which we were able to prevent the development of severe gastrointestinal disorders after capecitabine administration.

\section{Case Report}

The patient was a 64-year-old man who underwent high anterior resection for rectal cancer at a previous hospital. The cancer was a well-differentiated adenocarcinoma (pT3, pN1a, cM0, pStage IIIB, UICC TNM Classification, 8th Edition) and was treated with postoperative adjuvant chemotherapy consisting of capecitabine (oral FP, $2,000 \mathrm{mg} / \mathrm{m}^{2} /$ day, days 1 to 14) and oxaliplatin $\left(130 \mathrm{mg} / \mathrm{m}^{2}\right.$ on day 1) every 3 weeks. On chemotherapy initiation, he had an Eastern Cooperative Oncology Group (ECOG) performance

${ }^{1}$ Department of Gastroenterology, Faculty of Medicine, University of Tsukuba, Japan and ${ }^{2}$ Department of Gastrointestinal Surgery, Tsukuba medical center hospital, Japan

Received: September 5, 2021; Accepted: December 7, 2021; Advance Publication by J-STAGE: February 1, 2022

Correspondence to Dr. Yoshiyuki Yamamoto, y-yamamoto@md.tsukuba.ac.jp 
status (PS) of 0, no organ dysfunction, and no postoperative complications. On day 8, no hematopenia or nonhematologic toxicity was observed. On day 11 , he developed diarrhea, oral mucositis, anorexia, and a fever (all grade 1 according to the Common Terminology Criteria for Adverse Events version 5.0 [CTCAE v5.0]); these symptoms gradually worsened to grade 2-3. However, he did not contact the hospital and continued taking capecitabine until day 14 by his own decision.

On day 19, he developed grade 3 diarrhea, grade 3 renal dysfunction, and grade 3 febrile neutropenia (FN) (neutrophils, $380 / \mathrm{mm}^{3}$ ) and was urgently admitted to the previous hospital. Granulocyte colony-stimulating factor and meropenem were administered as an intravenous antibiotic treatment for FN. Later, he developed grade 4 renal dysfunction and was placed on temporary dialysis. He had continuous grade 3 diarrhea after admission and had bloody stool on day 28. An endoscopic examination revealed gastric ulcers and mucosal damage in the colon. It was judged that further treatment for systemic management would be difficult, so he was transferred to our hospital on day 46 for a detailed examination and intensive treatment.

DPD deficiency was suspected because he had experienced the early onset of many grade $\geq 3$ adverse events during the first cycle of chemotherapy at the previous hospital. Upon admission to our hospital, the DPD activity in peripheral blood mononuclear cells (PBMCs) was measured. His level of DPD activity was below the detection limit $(<1.06$ U/mg protein); therefore, he was diagnosed with DPD deficiency.

On admission, he had an ECOG PS of 3. A physical examination revealed a fever $\left(38.5^{\circ} \mathrm{C}\right)$, anemia of the palpebral conjunctiva, and grade 3 oral mucositis. The blood test data are presented in Table 1 . High levels of inflammatory markers, severe anemia, and low platelet count were observed; therefore, antibiotherapy and blood transfusion were initiated.

On day 46, esophagogastroduodenoscopy (EGD) revealed esophageal mucosal shedding (Fig. 1a) and extensive ulcers with contact bleeding in the lesser curvature of the gastric body (Fig. 1e). The exposed blood vessels at the bottom of the ulcer were coagulated to stop the bleeding. On day 47 , double-balloon endoscopy (DBE) revealed shedding of the ileal mucosa and multiple ulcers (Fig. 2a) and shallow mucosal shedding in the sigmoid colon (Fig. 2d). The bleeding was caused by persistent bleeding from multiple sites due to gastrointestinal mucosal disorders. Endoscopic hemostasis was difficult, and blood transfusion therapy was continued.

Endoscopy examinations were performed periodically. Fig. 1 shows the course of the EGD findings. The mucosa of the esophagus had fallen off circumferentially, but the healing process with fibrosis formation of the mucosa was observed (Fig. 1a-c). However, with improvement, segmental esophageal stenosis occurred, and the passage of the endoscope became impossible on day 109 (Fig. 1d). Extensive ulceration was observed in the lesser curvature of the gastric body; however, the ulcer tended to shrink (Fig. 1e-g). Fig. 2 shows the course of the colonoscopy (CS) findings. The ulcers in the terminal ileum showed regenerative epithelialization of the mucosa over time and tended to heal (Fig. 2a-c). Initially, multiple ulcers were found in the colon, and the mucosal damage tended to improve gradually (Fig. 2d-f). However, multiple stenoses occurred, and the endoscope could not be inserted into the mouth side of the transverse colon (Fig. 2g).

Fig. 3 shows the clinical course of the patient. Renal dysfunction and inflammatory responses improved over time. Although he suffered from hemorrhagic shock twice, his hemodynamics were stabilized by appropriate blood transfusions. The gastrointestinal mucosa eventually regenerated, and the bloody stool stopped on day 69. He had received a total of 184 units of red cell concentrate (RCC), 360 units of platelet concentrate (PC), and 92 units of fresh-frozen plasma (FFP) transfusions within the period up to day 67. After the bloody stool stopped, he still had severe diarrhea, which was suspected of being caused by protein leak syndrome and was treated with albumin supplementation and octreotide.

He was transferred to a rehabilitation hospital on day 161. $\mathrm{He}$ is still alive without any recurrence eight months after rectal cancer resection and continues to receive both central venous and enteral nutrition due to gastrointestinal transit and absorption problems.

\section{Discussion}

DPD is widely expressed in human tissues, and the liver is the major source of this enzyme; that is, with $80 \%$ of the total body supply (5). DPD activity is also high in PBMCs, with a comparable pattern to that in the liver. Therefore, PBMCs are widely used to measure DPD activity (6). A DPD activity of less than $30 \%$ is considered partially deficient, and that less than $10 \%$ is considered completely deficient. The proportions of partial DPD deficiency and complete DPD deficiency were reportedly $3 \%-5 \%$ and $0.1 \%$ in Caucasian and African American populations, respectively (7-10). However, detailed reports on DPD activity in Japan are scarce. The average DPD activity in 150 Japanese healthy volunteers was reported to be $173 \mathrm{U} / \mathrm{mg}$ protein; however, there were large individual differences (11).

Several methods of detecting DPD deficiency have been reported. DPD activities in PBMCs may be used as a marker for DPD activity in general $(6,8)$. Another method, involving the measurement of dihydrouracil and uracil in urine, is simple and useful but is associated with challenges for screening carriers and partial deficiency (12). The 2-13 C-uracil breath test rapidly discriminates between normal, partially, and profoundly DPD-deficient individuals, making it a useful screening method that can be used in most clinical settings $(13,14)$. However, its practicality remains unclear. Therefore, the measurement of DPD activity values in PBMC would be useful in Japan. 
Table 1. Laboratory Data at the Time of Admission to Our Hospital.

\begin{tabular}{lcllll}
\hline \multicolumn{2}{c}{ (Blood cell count) } & \multicolumn{2}{c}{ (Biochemistry) } & \multicolumn{2}{c}{ (Coagulation test) } \\
\hline WBC & $\underline{15.800} / \mu \mathrm{L}$ & $\mathrm{TP}$ & $\underline{4.1} \mathrm{~g} / \mathrm{dL}$ & $\mathrm{PT}$ & $\underline{55.7} \%$ \\
seg & $94 \%$ & Alb & $\underline{2.3} \mathrm{~g} / \mathrm{dL}$ & PT-INR & $\underline{1.42}$ \\
lym & $5 \%$ & T-Bil & $\underline{3.2} \mathrm{mg} / \mathrm{dL}$ & APTT & $\underline{44.2} \mathrm{sec}$ \\
mono & $1 \%$ & D-Bil & $\underline{2.2} \mathrm{mg} / \mathrm{dL}$ & FDP & $\underline{7.8} \mu \mathrm{g} / \mathrm{mL}$ \\
eos & $0 \%$ & AST & $25 \mathrm{U} / \mathrm{L}$ & & \\
RBC & $\underline{201 \times 10^{4}} / \mu \mathrm{L}$ & ALT & $17 \mathrm{U} / \mathrm{L}$ & \\
$\mathrm{Hb}$ & $\underline{6} \mathrm{~g} / \mathrm{dL}$ & $\mathrm{LDH}$ & $155 \mathrm{U} / \mathrm{L}$ & \\
$\mathrm{MCV}$ & $83.6 \mathrm{Fl}$ & $\Gamma$-GTP & $37 \mathrm{U} / \mathrm{L}$ & \\
$\mathrm{MCHC}$ & $35.7 \%$ & BUN & $\underline{60.8} \mathrm{mg} / \mathrm{dL}$ & \\
Plt & $\underline{5.0 \times 10^{4}} / \mu \mathrm{L}$ & $\mathrm{Cre}$ & $\underline{4.72} \mathrm{mg} / \mathrm{dL}$ & \\
& & $\mathrm{CRP}$ & $\underline{7.76} \mathrm{mg} / \mathrm{dL}$ & \\
\hline
\end{tabular}

WBC: white blood cell, RBC: red blood cell, Hb: hemoglobin, MCV: mean corpuscular volume, MCHC: mean corpuscular hemoglobin concentration, Plt: platelet, TP: total protein, Alb: albumin, T-Bil: total bilirubin, D-Bil: direct bilirubin, AST: aspartate aminotransferase, ALT: alanine aminotransferase, LDH: lactate dehydrogenase, $\Gamma$-GTP: $\Gamma$-glutamyl transpeptidase, BUN: blood urea nitrogen, Cre: creatinine, CRP: C-reactive protein, PT: prothrombin time, PT-INR: prothrombin time-international normalized ratio, APTT: activated partial prothrombin time, FDP: fibrinogen degradation products Underbar means abnormal value of the data.
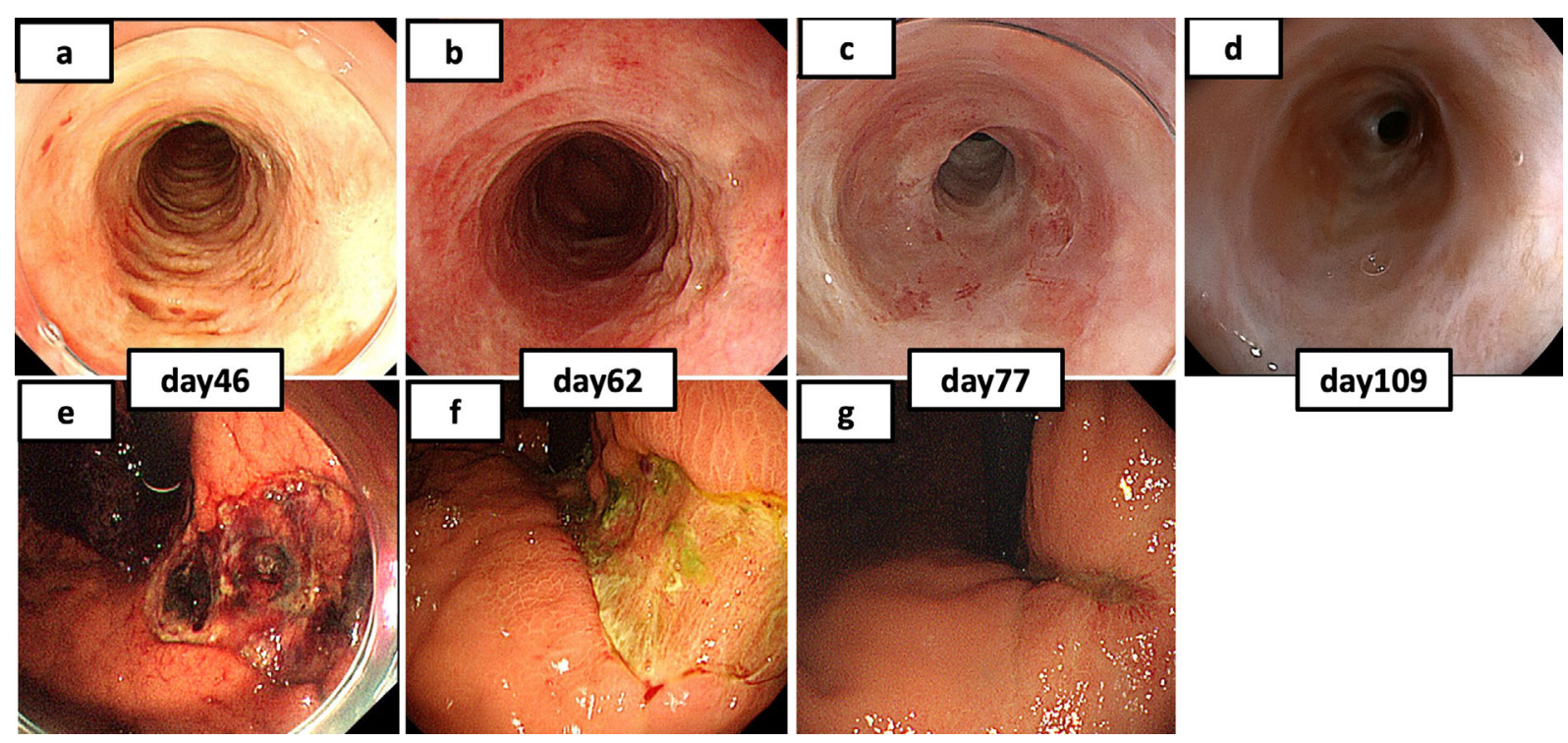

Figure 1. The course of mucosal damage findings on esophagogastroduodenoscopy. The mucosa of the esophagus had fallen off after capecitabine initiation (a). Gradual regeneration of the normal mucosa was observed (b). With improvement, stenosis occurred, and the endoscope could not be passed (c and d). Extensive ulceration was observed in the lesser curvature of the gastric body (e). The ulcer tended to shrink with the passage of time (f and g).

DPD deficiency is caused by alterations in the DPD gene. The DPD gene is mapped to chromosome 1p22 and consists of 23 exons $(15,16)$. The Clinical Pharmacogenetics Implementation Consortium guidelines stratify patients based on their DPD genotype and provide dosage recommendations according to their score (17). However, not all genetic variants have been identified, so data on more cases need to be collected.

In Japan, a total of 24 cases (including the present case) of severe adverse events were reported among patients with
DPD deficiency who were treated with FPs, according to a search using PubMed and a domestic article search engine $(3-5,18-37)$. The characteristics of these patients are summarized in Table 2. A total of 13 men and 11 women were reported, and the median age was 64.5 (range: 39-78) years old. Eleven patients had colorectal cancer, one had jejunum cancer, seven had gastric cancer, and five had breast cancer. Thirteen patients reported since 2012 received oral capecitabine. The main initial symptoms were gastrointestinal toxicities, accounting for $75 \%$ of the cases, which 


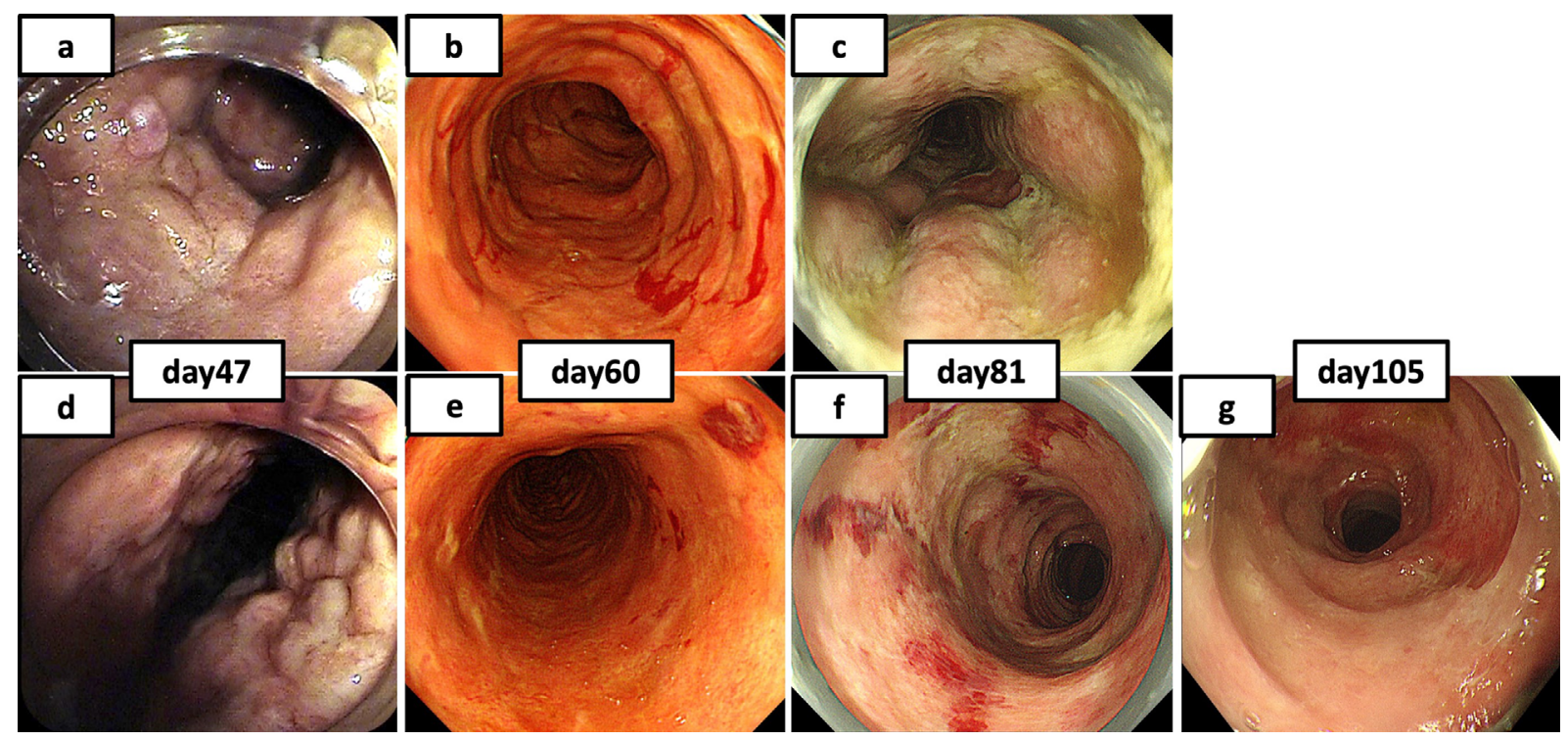

Figure 2. The course of mucosal damage findings in double-balloon endoscopy (a, d) and colonoscopy (b, c, e-g). The ulcers in the ileum showed regenerative epithelialization of the mucosa with the passage of time and tended to heal (a-c). Multiple ulcers were found in the colon (d). The mucosal damage tended to improve gradually (e-f). Stenosis occurred with the improvement, and the endoscope could not be inserted into the mouth of the transverse colon (g).
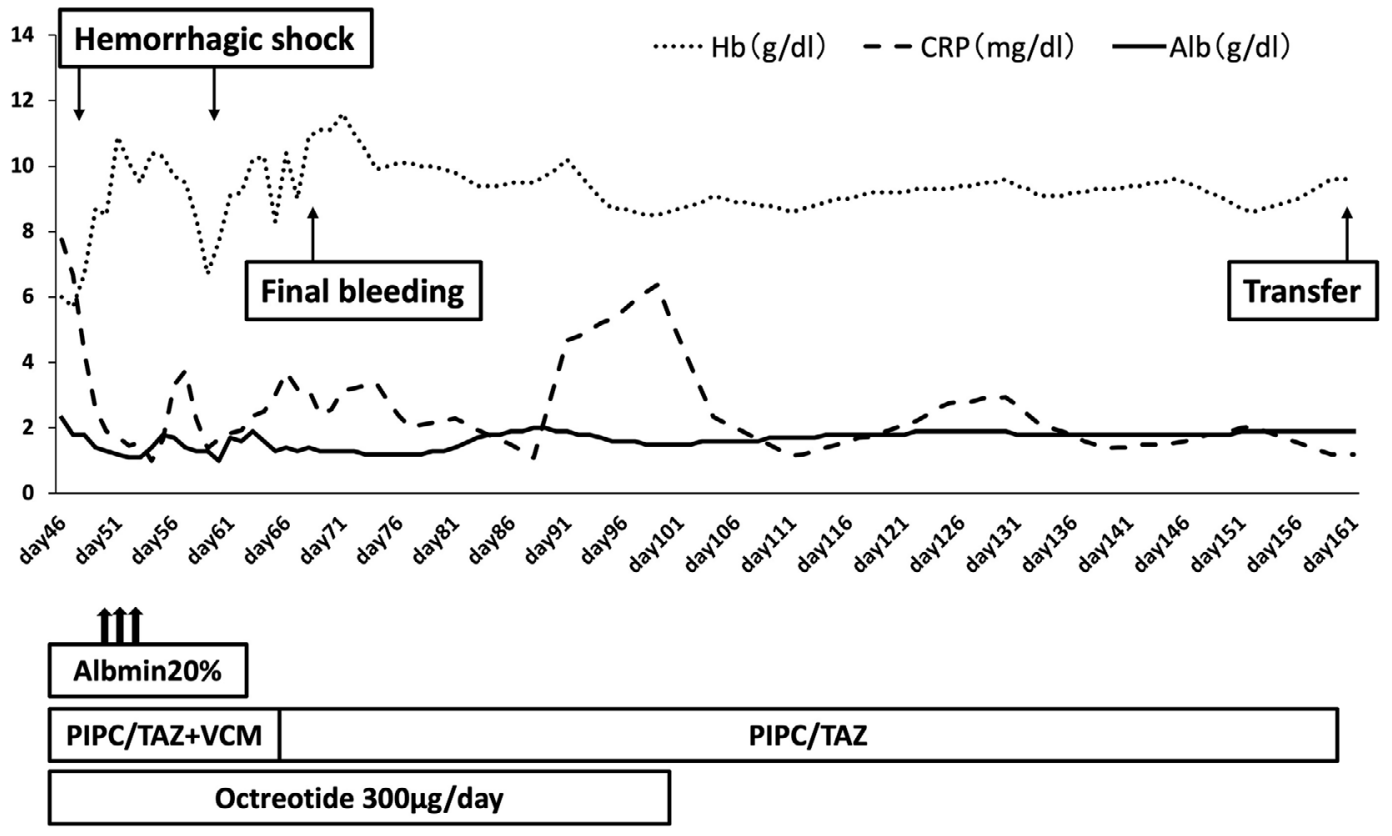

Figure 3. Clinical course after admission to our hospital. Serial changes in the levels of albumin, hemoglobin, CRP, and creatine, and the course of the administration of blood transfusions, intravenous therapeutics, and antibiotics are presented. Alb: albumin, Hb: hemoglobin, CRP: C-reactive protein, PIPC/TAZ: piperacillin/tazobactam, VCM: vancomycin

tended to appear earlier than hematologic toxicities. Most patients were diagnosed with DPD deficiency by measuring the DPD activity in the PBMCs. For the 15 patients with available DPD activity data, the average value was 7.97 (range: $0.32-21.5$ ) $\mathrm{U} / \mathrm{mg}$ protein. The mortality rate due to adverse events was approximately $29 \%$; specifically, it was $14 \%$ for the 7 patients with DPD activity $\geq 8 \mathrm{U} / \mathrm{mg}$ protein and $38 \%$ for the 8 patients with DPD activity $<8 \mathrm{U} / \mathrm{mg}$ protein, suggesting that a lower DPD activity may be associated with severe adverse events.

In foreign reports, most patients with DPD deficiency received 5-FU for colorectal cancer, and only few patients received oral FPs, including capecitabine (38). There have been several reports of severe gastrointestinal disorders in 
Table 2. Clinical Features of Severe Adverse Events in Japanese Patients with DPD Deficiency who Received Fluoropyrimidine.

\begin{tabular}{|c|c|c|}
\hline Sex, $\mathrm{n}$ & Male/Female & $13 / 11$ \\
\hline Age, years & Median (range) & $64.5(39-78)$ \\
\hline Cancer type, $\mathrm{n}$ & $\mathrm{CC} / \mathrm{RC} / \mathrm{JC} / \mathrm{GC} / \mathrm{BC}$ & $4 / 7 / 1 / 7 / 5$ \\
\hline Fluoropyrimidines, $\mathrm{n}$ & 5-FU / S-1 / UFT / Cape / 2 drugs (S-1 $\rightarrow$ Cape, S-1+UFT) & $6 / 1 / 3 / 12 / 2$ \\
\hline Onset day of gastrointestinal symptoms, day & Median (range) & $10(2-42)$ \\
\hline Onset day of blood toxicity, day & Median (range) & $14(5-42)$ \\
\hline Initial symptom*, $\mathrm{n}$ & gastrointestinal symptoms / oral mucositis / fever / hand-foot syndrome / n.d. & $18 / 4 / 2 / 1 / 1$ \\
\hline Diagnosis $^{\dagger}, \mathrm{n}$ & DPD activity (PMBC) / Uracil in urine / DPD gene variant / another & $22 / 6 / 3 / 1$ \\
\hline DPD protein amount ${ }^{\ddagger}, \mathrm{U} / \mathrm{mg}$ protein & Average (range) & $7.92(0.32-21.5)$ \\
\hline Prognosis, $\mathrm{n}$ & Recover / Death & $17 / 7$ \\
\hline Mortality according to DPD amount ${ }^{\ddagger}, \mathrm{n}$ & $<8 \mathrm{U} / \mathrm{mg} / \geq 8 \mathrm{U} / \mathrm{mg}$ & $3 / 1$ \\
\hline
\end{tabular}

*, Includes cases with multiple symptoms. $\dagger$, Includes cases with multiple diagnoses. $\ddagger$, Among 15 patients with available DPD activity data. DPD: dihydropyrimidine dehydrogenase, CC: colorectal cancer, RC: rectal cancer, JC: jejunum cancer, GC: gastric cancer, BC: breast cancer, Cape: capecitabine, n.d.: not demonstrated, PBMC: peripheral blood mononuclear cells, UFT: tegafur/uracil

foreign countries as well (39-41). In all cases, severe gastrointestinal symptoms were observed within two weeks of FP initiation. Attention should thus be paid to the appearance of gastrointestinal symptoms as well as blood toxicities, regardless of race.

Of the 24 previously reported cases, only Inoue et al. reported a patient with severe gastrointestinal disorders with hemorrhagic shock caused by capecitabine administration (36). Their patient had extensive ulcerations at the end of the ileum and hemorrhaging from multiple sites. Because of the multiple bleeding sites and the frequent shockinducing massive bleeding, endoscopic treatment was unable to control the bleeding, and the terminal ileum was surgically resected by $80 \mathrm{~cm}$. No bloody stool was observed after intestinal resection, and the patient's general condition improved. In the present case, mucosal shedding with bleeding was observed throughout the gastrointestinal tract, and surgical treatment was considered impossible. Conservative treatments saved the patient's life; however, extensive mucosal fibrosis and multiple gastrointestinal stenoses were observed. In the future, balloon dilation and bowel resection will be needed in order to restore oral intake after protein-losing enteropathy has been resolved. However, there have been no reports of DPD-deficient patients with gastrointestinal stenosis following mucosal injury caused by FP anticancer agents. Thus, it is necessary to follow up on the gastrointestinal function.

Capecitabine is an oral FP anticancer drug, absorbed from the gastrointestinal tract and converted to 5-fluorouracil (5FU) through three specific enzymatic reactions in the liver and major tissues. Uracil-tegafur and $\mathrm{S}-1$ constitute 5-FU pro-drugs and DPD inhibitors (uracil and gimeracil), allowing a reduction in the absolute dose of 5-FU. Thus, adverse events due to DPD deficiency are more likely to occur in patients treated with capecitabine than uracil-tegafur and $\mathrm{S}$ 1. Nevertheless, there was a report of death in a DPDdeficient patient treated with S-1 (4); therefore, caution should be exercised when administering any of these drugs.

Uridine triacetate competitively inhibits the incorporation of 5-FU into RNA and is effective in the treatment of fatal adverse events caused by overdose or oversensitivity to 5FU or capecitabine. It was approved by the U.S. Food and Drug Administration in December 2015 and is indicated for the treatment of severe adverse events occurring within $96 \mathrm{~h}$ of therapy initiation. There is a report of a patient with DPD deficiency who was saved by administration of uridine triacetate more than $96 \mathrm{~h}$ after the start of capecitabine administration, although it was used in an off-label capacity (42). It is important for this drug to be approved in Japan, although patients with DPD deficiency are rare.

Our patient continued to take capecitabine after the onset of symptoms. If the patient had been thoroughly educated to stop taking the drug early after the appearance of several symptoms and to seek medical attention, severe adverse events may have been able to be avoided. In addition, telephone follow-up has been reported to lead to a decrease in adverse events, which may be useful in similar cases (43). The measurement of DPD activity prior to the use of FP anticancer drugs may prevent the occurrence of serious adverse events. In Europe, the Pharmacovigilance Risk Assessment Committee recommended that phenotype and/or genotype testing for DPD deficiencies be performed prior to the administration of FPs. However, the frequency of DPD deficiency is extremely low in Japan, and it is not realistic to perform this procedure for all patients for cost-effective reasons. In Japan, telephone follow-up may be a feasible and effective means of reducing adverse events due to DPD deficiency. It is known that the DPD gene is inherited in an autosomal recessive manner, so it may be worthwhile to measure DPD activity in close relatives when FP anticancer drugs are used.

In conclusion, we can save patients with DPD-deficiency from a fatal status. However, it remains unclear whether or not the gastrointestinal function would improve. Therefore, in patients with DPD deficiency who present with early severe adverse events, such as hematologic and gastrointestinal toxicities, it is important to discontinue FP and prevent their aggravation.

The authors state that they have no Conflict of Interest (COI). 


\section{Acknowledgement}

Funding: This research did not receive any specific grants from any funding agencies in the public, commercial, or not-forprofit sectors. We received support from Chugai Pharmaceutical for the measurement of DPD activity in PBMCs.

Authors' Contributions: All authors were equally involved in the patients' treatment, drafting of the manuscript, and approval of the final manuscript.

\section{References}

1. Lu Z, Zhang R, Diasio RB. Purification and characterization of dihydropyrimidine dehydrogenase from human liver. J Biol Chem 267: 17102-17109, 1992.

2. Milano G, Etienne MC. Dihydropyrimidine dehydrogenase (DPD) and clinical pharmacology of 5-fluorouracil. Anticancer Res 14: 2295-2297, 1994.

3. Takaba T, Moriyama J, Yokoyama T, Matoba S, Sawada T. A case of rectal cancer with dihydropyrimidine dehydrogenase deficiency. Jpn J Gastroenterol Surg 41: 2075-2080, 2008.

4. Aragane H, Suchi K, Shimomura M, Katano T, Yasui H, Kan K. Severe bone marrow suppression during adjuvant chemotherapy for gastric cancer by S-1 and its possible relationship to dihydropyrimidine dehydrogenase deficiency. Gan To Kagaku Ryoho 37: 131-133, 2010 (in Japanese).

5. Matsumoto A, Fujita T, Ozaki T, et al. Dihydropyrimidine dehydrogenase deficiency with severe adverse events caused by XELOX + Bevacizumab. Jpn J Gastroenterol Surg 47: 734-739, 2014 (in Japanese).

6. Chazal M, Etienne MC, Renée N, Bourgeon A, Richelme H, Milano G. Link between dihydropyrimidine dehydrogenase activity in peripheral blood mononuclear cells and liver. Clin Cancer Res 2: 507-510, 1996.

7. Fleming RA, Milano GA, Gaspard MH, et al. Dihydropyrimidine dehydrogenase activity in cancer patients. Eur J Cancer 29A: 740744, 1993.

8. Lu Z, Zhang R, Diasio RB. Dihydropyrimidine dehy-drogenase activity in human peripheral blood mononu-clear cells and Iiver: population characteristics,newly identified deficient patients, and clinical implication in 5-fluorouracil chen otherapy. Cancer Res 53: 5433-5438, 1993.

9. Etienne MC, Lagrange JL, Dassonville O, et al. Population study of dihydropyrimidine dehydrogenase in cancer patients. J Clin Oncol 12: 2248-2253, 1994

10. Lu Z, Zhang R, Diasio MD. Population characteristics of hepatic dihydropyrimidine dehydrogenase activity, a key metabolic enzyme in 5-fluorouracil chemotherapy. Clin Pharmacol Ther 58: 512-522, 1995.

11. Ogura K, Ohnuma T, Minamide $Y$, et al. Dihydropyrimidine DehydrogenaseActivity in150 HealthyJapanese Volunteers and Identification of NovelMutations. Human Cancer Biology 11: 5104-5111, 2005.

12. Hayashi K, Kidouchi K, Sumi S, et al. Possible prediction of adverse reactions to pyrimidine chemotherapy from urinary pyrimidine levels and a case of asymptomatic adult dihydropyrimidinuria. Clin Cancer Res 2: 1937-1941, 1996.

13. Mattison LK, Ezzeldin H, Carpenter M, et al. Rapid identification of dihydropyrimidine dehydrogenase deficiency by using a novel 2-13C-uracil breath test. Clin Cancer Res 10: 2652-2658, 2004.

14. Mattison LK, Fourie J, Hirao Y, Koga T, et al. The uracil breath test in the assessment of dihydropyrimidine dehydrogenase activity: pharmacokinetic relationship between expired $13 \mathrm{CO} 2$ and plasma [2-13C] dihydrouracil. Clin Cancer Res 12: 549-555, 2006.
15. Deenen MJ, Meulendijks D, Cats A, et al. Upfront Genotyping of DPYD*2A to Individualize Fluoropyrimidine Therapy. A Safety and Cost Analysis. J Clin Oncol 34: 227-234, 2016.

16. Boige V, Vincent M, Alexandre P, et al. DPYD Genotyping to Predict Adverse Events Following Treatment With FluorouracilBased Adjuvant Chemotherapy in Patients With Stage III Colon Cancer A Secondary Analysis of the PETACC-8 Randomized Clinical Trial. Jama Oncology 2: 655-662, 2016.

17. Gentile G, Botticelli A, Lionetto L, et al. Genotype-phenotype correlations in 5-fluorouracil metabolism: a candidate DPYD haplotype to improve toxicity prediction. Pharmacogenomics J 16: 320$325,2016$.

18. Kouwaki M, Hamajima N, Sumi S, et al. Identification of novel mutations in the dihydropyrimidine dehydrogenase gene in a Japanese patient with 5-fluorouracil toxicity. Clin Cancer Res 4: 29993004, 1998.

19. Kobayashi K, Sumi S, Kidouchi K, et al. A case of gastric cancer with decreased dihydropyrimidine dehydrogenase activity. Gan To Kagaku Ryoho 25: 1217-1219, 1998 (in Japanese).

20. Inada T, Jotsuka T, Matsuda G, Kawakubo H, Ogata Y, Kubota T. Severe 5-fluorouracil-related toxicity in a Japanese patient with dihydropyrimidine dehydrogenase deficiency. Int J Clin Oncol 4: 5456, 1999.

21. Sumi S, Kidouchi K, Imaeda M, et al. Side effect of fluorouracil system anticancer drug and dysbolism of pyrimidine. Rinshoyakuri no Shinpo 2261-2266, 2001 (in Japanese).

22. Godai T, Shimizu T, Kinjo M. A case of DPD deficiency with severe side effects of 5-FU. Jpn J Gastroenterol Surg 63: 698, 2002 (in Japanese).

23. Komuro Y, Mikoshiba M, Sasaki E, et al. A case of DPD deficiency associated with severe intestinal mucosal damage and myelosuppression after 5-FU treatment with outcome of death. Jpn Oral Oncology 39: 747, 2004 (in Japanese).

24. Hashimoto T, Arai K, Iwasaki Y, et al. A case of recurrent gastric cancer with dihydropyrimidine dehydrogenase (DPD) deficiency. Gan To Kagaku Ryoho 33: 985-988, 2006 (in Japanese).

25. Kai K, Endo Y, Yoshida K, et al. A case of dihydropyrimidine dehydrogenase (DPD) deficiency with severe side effects from UFT/ Uzel administration. Gan To Kagaku Ryoho 35: 339-341, 2008 (in Japanese).

26. Iwamoto A, Kishi $K$, Takemoto $H$, Nishie $H$, Maeta M. A case of DPD low activity with severe fluorouracil toxicity caused by UFT/ Uzel. J Jpn Surg Assoc 71: 2791-2794, 2010 (in Japanese).

27. Tomioka Y, Yoshimura H. A case of suspected DPD deficiency with severe side effects after treatment with capecitabine for bone metastasis of breast cancer. Program and Abstracts of the Annual Meeting of the Japanese Breast Cancer Association 20: 455, 2012 (in Japanese).

28. Tsukiyama G, Hasegawa M, Yabuki S, Tanaka H, Tanahashi C. A case of good response in a rectal cancer patient with decreased dihydropyrimidine dehydrogenase activity because of strict control of the 5-fluorouracil dose. Gan To Kagaku Ryoho 40: 2023-2025, 2013 (in Japanese).

29. Sakaguchi H, Miyamoto H, Ono K, Saito T, Gomyo Y, Ikeno T. A case of suspected dihydropyrimidine dehydrogenase (DPD) deficiency in which severe adverse events occurred during postoperative adjuvant chemotherapy with capecitabin. Rinshogeka 69: 617620, 2014 (in Japanese).

30. Nagai K, Okuda Y, Ohara Y, Yamamoto M. Suspected dihydropyrimidine dehydrogenase deficiency in a patient receiving capecitabine as adjuvant chemotherapy after colon resection. Gan To Kagaku Ryoho 42: 127-129, 2015 (in Japanese).

31. Kinoshita $H$, Iwamoto $H$, Umano $Y$, Tsubakihira $H$, Sakata $Y$, Mori K. A case of cytomegalovirus colitis that developed during chemotherapy for advanced gastric cancer with low activity of dihydropyrimidine dehydrogenase. J Jpn Surg Assoc 76: 1020-1024, 
2015 (in Japanese).

32. Mitake Y, Hiramatsu K, Kato T, Shibata Y, Yoshihara M, Aoba T. A case of low dihydropyrimidine dehydrogenase activity with septic shock caused by enterocolitis during adjuvant chemotherapy for stage II colon cancer. J Jpn Surg Assoc 76: 2247-2251, 2015 (in Japanese)

33. Yoshida Y, Ogura K, Hiratsuka A, et al. 5-Fluorouracil Chemotherapy for Dihydropyrimidine Dehydrogenase-deficient Patients: Potential of the Dose-escalation Method. Anticancer Res 35: 48814887, 2015.

34. Sakata H, Shimizu E, Fujita K, Yamaguchi Y, Suzuki T, Matsubara H. Low activity of dihydropyrimidine dehydrogenase associated with severe adverse effects after administration of adjuvant CapeOX in the treatment of rectal cancer. J Jpn Surg Assoc 78: 1207-1212, 2017 (in Japanese).

35. Watanabe H, Arita S, Takeuchi T, Oshima Y, Koike N. A Case of Colon Cancer with DPD Deficiency That Showed Severe Myelosuppression by CapeOX Adjuvant Chemotherapy after Colon Resection. Gan To Kagaku Ryoho 45: 1661-1664, 2018 (in Japanese).

36. Inoue H, Sato Y, Shintani S, et al. Dihydropyrimidine dehydrogenase deficiency causes severe adverse effects of capecitabine. Nihon Shokakibyo Gakkai Zasshi 115: 290-298, 2018 (in Japanese).

37. Ishiguro M, Takenaka R, Ogura K. A Japanese Patient with Gastric Cancer and Dihydropyrimidine Dehydrogenase Deficiency Presenting with DPYD Variants. Acta media Okayama 74: 557$562,2020$.

38. Stephanie D, Eva S, Marzia DR, et al. Dihydropyrimidine dehydrogenase deficiency in patients with severe toxicity after 5fluorouracil: a retrospective single-center study. Annals of Gastro- enterology 34: 68-72, 2021.

39. Ezzeldin H, Johnson MR, Okamoto Y, et al. Denaturing high performance liquid chromatography analysis of the DPYD gene in patients with lethal 5-fluorouracil toxicity. Clin Cancer Res 9: 3021-3028, 2003.

40. Mounier-Boutoille H, Boisdron-Celle M, Cauchin E, et al. Lethal outcome of 5-fluorouracil infusion in a patient with a total DPD deficiency and a double DPYD and UTG1A1 gene mutation. Br J Clin Pharmacol 70: 280-283, 2010.

41. van Kuilenburg AB, Muller EW, Haasjes J, et al. Lethal outcome of a patient with a complete dihydropyrimidine dehydrogenase (DPD) deficiency after administration of 5-fluorouracil: frequency of the common IVS14+1G\&A mutation causing DPD deficiency. Clin Cancer Res 7: 1149-1153, 2001.

42. Mira Z, Yi-Kong K, David Y, et al. Successful use of uridine triacetate (Vistogard) three weeks after capecitabine in a patient with homozygous dihydropyrimidine dehydrogenase mutation: A case report and review of the literature. J Oncol Pharm Practice 25: 234-238, 2019.

43. Hend KE, Maggie MA, Marwa MH, et al. Telephone-Based Follow-Up on Adherence, Efficacy, and Toxicity of Oral Capecitabine-Based Chemotherapy. Telemed J E Health 25: 462470, 2019.

The Internal Medicine is an Open Access journal distributed under the Creative Commons Attribution-NonCommercial-NoDerivatives 4.0 International License. To view the details of this license, please visit (https://creativecommons.org/licenses/ by-nc-nd/4.0/).

(C) The Japanese Society of Internal Medicine Intern Med Advance Publication 